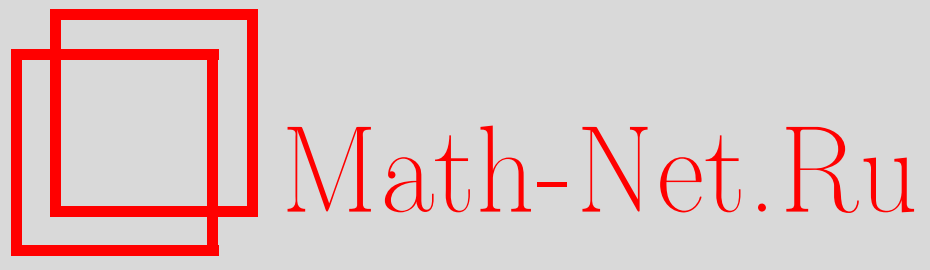

Л. В. Розовский, Малые уклонения взвешенной суммы независимых положительных случайных величин с общим распределением, убывающим в нуле не быстрее степени, Теория вероятн. и ее примен., 2015, том 60, выпуск 1, 178-186

DOI: https://doi.org/10.4213/tvp4613

Использование Общероссийского математического портала Math-Net.Ru подразумевает, что вы прочитали и согласны с пользовательским соглашением http: //www . mathnet.ru/rus/agreement

Параметры загрузки:

IP : 54.196 .121 .252

26 апреля 2023 г., $18: 10: 48$

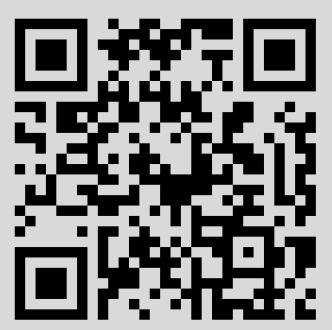


(c) 2015 г.

РОзОВСКИЙ Л. В.*

\title{
МАЛЫЕ УКЛОНЕНИЯ ВЗВЕШЕННОЙ СУММЫ НЕЗАВИСИМЫХ ПОЛОЖИТЕЛЬНЫХ СЛУЧАЙНЫХ ВЕЛИЧИН С ОБЩИМ РАСПРЕДЕЛЕНИЕМ, УБЫВАЮЩИМ В НУЛЕ НЕ БЫСТРЕЕ СТЕПЕНИ ${ }^{1)}$
}

\begin{abstract}
В заметке показано, что известная асимптотика для вероятностей малых уклонений взвешенной суммы независимых положительных случайных величин с общим распределением остается справедливой, если это распределение в нуле убывает не быстрее некоторой степени.
\end{abstract}

Ключевые слова и фразы: малые уклонения, суммы независимых положительных случайных величин, медленно меняющиеся функции.

1. Введение и результаты. Пусть $\left\{X_{i}\right\}-$ независимые копии положительной случайной величины $X$ с функцией распределения $V(x)$. Образуем $S=\sum_{j \geqslant 1} \lambda_{j} X_{j}$, где $\left\{\lambda_{j}\right\}$ - некоторая последовательность невозрастаюших положительных чисел. Всюду в дальнейшем нами предполагается, что ряд $S$ сходится п.н. или, равносильно (см., например, [3]),

$$
\sum_{j \geqslant 1} \mathbf{E}\left(1 \wedge \lambda_{j} X\right)<\infty
$$

Заметим, что если $\mathbf{E} X<\infty$, то предположение (1.1) совпадает с условием $\sum_{j} \lambda_{j}<\infty$.

3 а м е ч а н и е 1 . Пусть функция $b(y), y \geqslant y_{0}$, не убывает и удовлетворяет условию $b\left(1 / \lambda_{j}\right) \asymp j, j \rightarrow \infty$. Тогда (1.1) равносильно условию $\mathbf{E} \bar{b}(X) I\left[X>y_{0}\right]<\infty$, где

$$
\bar{b}(y)=y \int_{y}^{\infty} \frac{b(u)}{u^{2}} d u, \quad y>y_{0} .
$$

Отметим, что

$$
1 \leqslant \frac{\bar{b}(y)}{b(y)} \leqslant \int_{1}^{\infty} \sup _{y>y_{0}} \frac{b(u y)}{b(y)} \frac{d u}{u^{2}} .
$$

В частности, если $b(u) / u^{1-\delta}$ при некотором $\delta>0$ убывает на бесконечности, то (1.1) равносильно $\mathbf{E} b(X) I\left[X>y_{0}\right]<\infty$.

Для $\gamma \geqslant 0$ положим

$$
\begin{gathered}
\Lambda(\gamma)=\mathbf{E} e^{-\gamma S}, \quad m(\gamma)=-(\ln \Lambda(\gamma))^{\prime}, \quad \sigma^{2}(\gamma)=(\ln \Lambda(\gamma))^{\prime \prime}, \\
Q(\gamma)=-\gamma m(\gamma)-\ln \Lambda(\gamma) .
\end{gathered}
$$

В работе [1], уточняющей и обобщающей результаты многочисленных предшественников (см. ссылки в [1], а также [6]), было доказано, что при некоторых условиях, включающих предположение, что $V(r)$ ведет себя в нуле степенным образом (см. условие (L) ниже),

$$
\mathbf{P}(S \leqslant r)=(2 \pi)^{-1 / 2}(\gamma \sigma(\gamma))^{-1} e^{-Q(\gamma)}(1+o(1)), \quad r \searrow 0,
$$

где параметр $\gamma=\gamma(r)$ является решением уравнения $m(\gamma)=r$.

Исследования, предпринятые в [1], были продолжены в [2] и [3].

* С.-Петербургская химико-фармацевтическая академия, С.-Петербург, Россия; e-mail: l_rozovsky@mail.ru

1) Работа выполнена при поддержке РФФИ (грант № 13-01-00256-а) и программы «Ведущие научные школы» (грант НШ-2504.2014.1). 
В настоящей заметке нами рассмотрена более общая ситуация, включающая возможность для $V(r)$ убывать в нуле медленнее любой степени $r$ (например, быть медленно меняющейся в нуле функцией), и уточнен основной результат из [3].

Введем некоторые условия и обозначения.

Начнем с у с л о в и я $(\mathbf{L})$, предложенного в [1]: существуют постоянные $b \in$ $(0,1), c_{1}, c_{2}>1$ и $r_{0}>0$ такие, что при каждом $r \leqslant r_{0}$

$$
c_{1} V(b r) \leqslant V(r) \leqslant c_{2} V(b r),
$$

из которого, в частности, следует, что

$$
c_{3} r^{\alpha} \leqslant V(r) \leqslant c_{4} r^{\beta}, \quad 0<r \leqslant r_{0},
$$

где $\alpha=\ln d_{2} /|\ln a|$ и $\beta=\ln d_{1} /|\ln a|$, а $c_{3}, c_{4}, \ldots$ здесь и в дальнейшем обозначают положительные постоянные, зависящие лишь от распределения $V$.

Условие $(\mathbf{L})$ представляет собой широкое обобщение предположения о том, что распределение $\mathrm{V}$ является правильно меняющейся в нуле функцией с положительным показателем $\alpha$.

Нам понадобится также у с л о в и е $(\mathbf{F})$ :

$$
\limsup _{x \rightarrow \infty} \frac{x^{2} \mathbf{P}(X \geqslant x)}{\mathbf{E} X^{2} I[X<x]}<\infty .
$$

Напомним, что $(\mathbf{F})$ выполняется, когда $X$ принадлежит области притяжения какоголибо устойчивого закона, в частности, имеет конечную дисперсию.

В [3, теорема 2.3] был получен следующий результат.

Теорема 1. Если выполнень условия $(\mathbf{L})$ и $(\mathbf{F})$, то при всех положительных $r$, $s u \gamma$

$$
\begin{aligned}
& \mathbf{P}(r-s<S \leqslant r)=\Lambda(\gamma) e^{\gamma r} \frac{1-e^{-\gamma s}}{\tau \sqrt{2 \pi}} \\
& \quad \times\left(\operatorname { e x p } \left\{-\frac{(r-m(\gamma))^{2}}{2 \sigma^{2}(\gamma)}+\theta\left(\tau^{-1}+\left(\frac{\ln (1+\tau)}{\tau^{2}}\right)^{1 / \alpha}\left(1+\frac{1}{\gamma s}\right)\right\}\right.\right.
\end{aligned}
$$

где $\tau=\gamma \sigma(\gamma), \alpha=\ln c_{2} /|\ln b|, a|\theta| \leqslant c_{3}$.

Соотношение (1.3) (с оценкой остаточного члена) вытекает из теоремы 1 при $s=-\infty$ и $\gamma$, являющемся решением уравнения $m(\gamma)=r$, так как из условия $(\mathbf{L})$ следует, что

Для $y>0$ положим

$$
\gamma \sigma(\gamma) \rightarrow \infty, \quad \gamma \rightarrow \infty
$$

$$
\nu(y)=\int_{0}^{y} u d V(u), \quad \widehat{\nu}(y)=\frac{\nu(y)}{y}
$$

и введем у с л о в и е $(\mathbf{R}):$ существуют постоянные $b \in(0,1), c_{2}, c_{1}>1 u y_{0}>0$ такие, ито при каждом $y \leqslant y_{0}$

$$
c_{1} \nu(b y) \leqslant \nu(y) \leqslant c_{2} \nu(b y) .
$$

Отметим, что правое неравенство в (1.9) может выполняться лишь тогда, когда $c_{2} b>1$ (см. доказательство замечания 2 ниже).

Сравним условия $(\mathbf{L})$ и $(\mathbf{R})$.

З а м е ч а н и е 2. Справедливо утверждение

$$
\left.(\mathbf{L}) \Longleftrightarrow(\mathbf{R})\right|_{c_{1} b>1} \text {. }
$$

В то же время, если

$$
\widehat{\nu}(y) \asymp l(y), \quad y \rightarrow+0,
$$


где функция $l(y)$ медленно меняется в нуле, то

$$
V(y) \asymp \widetilde{l}(y)=\int_{0}^{y} \frac{l(u)}{u} d u, \quad y \rightarrow+0,
$$

причем $\widetilde{l}(y)$ также медленно меняется в нуле. Отсюда следует, что (1.9) при достаточно малом $b$ выполняется, в то время как условие (1.4) нарушается в левой его части. Таким образом, $(\mathbf{R})$ слабее $(\mathbf{L})$.

Заметим, что условия (1.10) и (1.11), очевидно, имеют место, если распределение $V$ абсолютно непрерывно в некоторой окрестности нуля с плотностью $p(x)$ такой, что $p(y) \asymp l(y) / y, y \rightarrow+0$.

Докажем замечание 2. Поскольку

$$
V(b y)=b^{-1}\left(\frac{\nu(b y)}{y}+\int_{0}^{y} \frac{\nu(b u)}{u^{2}} d u\right),
$$

из (1.9) следует (1.4) при $a=b, d_{1}=c_{1} b$ и $d_{2}=c_{2} b$, т.е. $\left.(\mathbf{R})\right|_{c_{1} b>1} \Longrightarrow(\mathbf{L})$. Кроме того, $V(b y) \leqslant V(y) \leqslant c_{2} b V(b y), y \leqslant y_{0}$, откуда с учетом того, что $\mathbf{P}(X=0)=0$, получим $c_{2} b>1$.

Пусть теперь выполнено условие $(\mathbf{L})$. Имеем $\widehat{\nu}(y) / V(y)=\int_{0}^{1}(1-V(u y) / V(y)) d u$. Отсюда, а также из вытекающего из (1.4) соотношения $d_{1} u^{\beta} \geqslant V(u y) / V(y) \geqslant u^{\alpha} / d_{2}$, $u \leqslant 1, y \leqslant y_{0}$ (см. [2, формула (1.8)] и обозначения после (1.5), из которых следует, что $\left.a^{\alpha}=1 / d_{2}, a^{\beta}=1 / d_{1}\right)$, найдем

$$
1-\frac{1}{(1+\alpha) d_{2}} \geqslant \frac{\widehat{\nu}(y)}{V(y)} \geqslant \int_{0}^{a}\left(1-d_{1} u^{\beta}\right) d u=\frac{a \beta}{1+\beta} .
$$

Из приведенных формул и (1.4) получим при любом $b \in(0, a]$

$$
c^{-1}(a b)^{1+\alpha} \leqslant \frac{\nu(b y)}{\nu(y)} \leqslant c\left(\frac{b}{a}\right)^{1+\beta}, \quad y \leqslant y_{0},
$$

где

$$
c=\left(1-\frac{1}{(1+\alpha) d_{2}}\right)\left(1+\frac{1}{\beta}\right) .
$$

Отсюда при достаточно малом $b$ следует $\left.(\mathbf{R})\right|_{c_{1} b>1}$. Замечание 2 проверено.

Теперь сформулируем основной результат настоящей работы.

Теорема 2. Теорема 1 сохраняет справедливость при замене в ее формулировке условия $(\mathbf{L})$ на $(\mathbf{R})$ и постоянной $\alpha н a \ln \left(c_{2} b\right) /|\ln b|$.

Заметим, что теорема 2 нетривиальна лишь в случае (1.7), который, как уже упоминалось выше, всегда имеет место при условии $(\mathbf{L})$, а из $(\mathbf{R})$, вообще говоря, не вытекает: в некоторых случаях

$$
\limsup _{\gamma \rightarrow \infty} \gamma \sigma(\gamma)<\infty
$$

Стоит также отметить, что обязательно $\lim \sup _{\gamma \rightarrow \infty} \gamma \sigma(\gamma)>0$.

Теорему 2 можно дополнить следующим результатом.

Теорема 3. Пусть выполнено условие (1.12). Тогда (см. обозначения в (1.2))

$$
\mathbf{P}(S \leqslant r) \asymp e^{-Q(\gamma)}, \quad r \searrow 0,
$$

где параметр $\gamma=\gamma(r)$ является решением уравнения $m(\gamma)=r$.

Заметим, что в теореме 3 выполнение условий $(\mathbf{R})$ или $(\mathbf{F})$ не предполагается.

3 а м е ч а н и е 3 . Пусть выполнено условие $(\mathbf{F})$. Если

$$
\lim _{M \rightarrow \infty} \liminf _{N \rightarrow \infty} \frac{\lambda_{N+M}}{\lambda_{N}}>0,
$$


то справедливо условие (1.7), причем без каких-либо предположений относительно поведения $V$ в нуле.

Отметим, что (1.13) выполнено, если

$$
\lambda_{j} \asymp e^{-g(j)}, \quad j \rightarrow \infty,
$$

где функция $g$ не убывает и

$$
\frac{g(u)}{u} \text { монотонно стремится к нулю на бесконечности. }
$$

Таким образом, в предположении (1.13) асимптотика (1.3) из теоремы 2 вытекает. Вернемся к условию (1.12).

3 а м е ч а н и е 4. Предположим, что выполнены условия (1.14) и (1.10) с медленно меняющейся в нуле функцией $l(y)$ такой (см. (1.11)), что $\widetilde{l}\left(e^{-t}\right)$ логарифмически выпукла на бесконечности.

Если $g(u), u \geqslant 1$, - некоторая непрерывная положительная функция такая, что

$$
\text { функция } \frac{g(u)}{u} \text { не убывает на бесконечности, }
$$

то условие

$$
J(u)=\frac{u}{g(u)}\left|\ln \widetilde{l}\left(e^{-g(u)}\right)\right|=O(1), \quad u \rightarrow \infty,
$$

является достаточным для (1.12), а если $g(u)$ не убывает на бесконечности и $g(u) / u^{K}$ убывает при всех достаточно больших $u$ и некотором $K$, то условие (1.17) является необходимым для (1.12). Кроме того, при таких функциях $g(u)$ из предположения $J(u) \rightarrow \infty, u \rightarrow \infty$, вытекает (1.7).

П р и м е р. Пусть $a>0$ и $c \geqslant 1-$ некоторые постоянные. Если

$$
\lambda_{j} \asymp \exp \left(-a j^{c}\right), \quad j \rightarrow \infty,
$$

то (1.12) в соответствии с замечанием 4 равносильно

$$
u^{1 / c-1}\left|\ln \widetilde{l}\left(e^{-u}\right)\right|=O(1), \quad u \rightarrow \infty .
$$

Таким образом, если, например, $l(y)=\ln ^{-1-\delta}(1 / y)$, где $\delta>0$, то при $c>1$ имеет место (1.12), а при $c=1$ справедливо (1.7).

Отметим также, что предположение (1.1), которое, разумеется, считается выполненным, в случае (1.18) при $c>0$ по замечанию 1 равносильно условию $\mathbf{E} \ln ^{1 / c}(1+X)<\infty$ и вытекает из условия $(\mathbf{F})$.

2. Доказательства. В нижеследующих рассуждениях существенно используются результаты из [2]-[4].

Пусть $L(h)=\mathbf{E} e^{-h X}$ и случайная величина $X(h), h \geqslant 0$, имеет распределение $e^{-h r} V(d r) / L(h)$ и характеристическую функцию $f_{h}(t)$.

Обозначим дополнительно (см. (1.8))

$$
\widetilde{\nu}(y)=\int_{0}^{y} \frac{\widehat{\nu}(u)}{u} d u, \quad \kappa(y)=\frac{\widehat{\nu}(y)}{\widetilde{\nu}(y)} .
$$

Лемма 1. Пусть выполнено условие $(\mathbf{R})$. Тогда при всех $h \geqslant 1$

$$
\begin{gathered}
c_{3} \widetilde{\nu}\left(\frac{1}{h}\right) \leqslant L(h), \quad V\left(\frac{1}{h}\right) \leqslant c_{4} \widetilde{\nu}\left(\frac{1}{h}\right), \\
c_{3} \kappa\left(\frac{1}{h}\right) \leqslant h \mathbf{E} X(h), \quad h^{2} \mathbf{D} X(h) \leqslant c_{4} \kappa\left(\frac{1}{h}\right), \\
\frac{h \mathbf{E} X^{3}(h)}{\mathbf{D} X(h)} \leqslant c_{4} .
\end{gathered}
$$


Кроме того,

$$
1-\left|f_{h}(v)\right| \geqslant c_{5} e^{-(2 \pi+b) h / v} \frac{\widehat{\nu}(1 / v)}{L(h)}, \quad v \geqslant \frac{2 \pi+b}{r_{0}}, \quad h>0 .
$$

Напомним, что $c_{3}, c_{4}, \ldots$ обозначают положительные постоянные, зависящие лишь от распределения $V$.

Д о к а з а т е л ь с т в о л е м м ы 1. Проверим (2.2)-(2.4). Пусть (см. (1.9))

$$
\nu(y) \leqslant c_{2} \nu(b y), \quad y \leqslant y_{0} .
$$

Тогда

$$
\widetilde{\nu}(y) \geqslant \frac{1-b}{b y} \nu(b y) \geqslant \frac{1-b}{b c_{2}} \widehat{\nu}(y)
$$

и, с учетом равенства $V(y)=\widehat{\nu}(y)+\widetilde{\nu}(y)$,

$$
\widetilde{\nu}(y) \leqslant V(y) \leqslant c_{4} \widetilde{\nu}(y), \quad y \leqslant y_{0} .
$$

Из (2.6) следует, что при всех $u \geqslant 1$

$$
\nu(u y) \leqslant c_{2} u^{\gamma} \nu(y), \quad u y \leqslant b y_{0},
$$

где $\gamma=\ln c_{2} /|\ln b|>1$.

Действительно, если натуральное $k$ удовлетворяет условию $b^{1-k} \leqslant u<b^{-k}$, то последовательное применение соотношения (2.6) $k$ раз приводит к (2.9). Неравенство $\gamma>1$ равносильно соотношению $c_{2} b>1$.

Используя (2.9) при $u=b y_{0} / y$, получим, в частности, что

$$
\nu(y) \geqslant \frac{\nu\left(b y_{0}\right)}{c_{2}\left(b y_{0}\right)^{\gamma}} y^{\gamma}, \quad y \leqslant b y_{0} .
$$

Имеем для $h \geqslant h_{0} \geqslant 1 /($ byo $)$ :

$$
e^{-1} V\left(\frac{1}{h}\right) \leqslant L(h)=\left(\int_{0}^{1}+\int_{1}^{b y_{0} h}+\int_{b y_{0} h}^{\infty}\right) \widehat{\nu}\left(\frac{u}{h}\right) e^{-u} \frac{1+u}{u} d u=I_{1}+I_{2}+I_{3} .
$$

При этом (см. также $(2.7)$ и $(2.10)) I_{1} \leqslant \widetilde{\nu}(1 / h), I_{3} \leqslant 2 e^{-b y_{0} h} \leqslant c_{5} \widetilde{\nu}(1 / h)$. Оценивая $I_{2}$ с помощью (2.9) при $y=b / h$ и применяя $(2.7)$, найдем $I_{2} \leqslant c_{6} \widehat{\nu}(b / h) \leqslant c_{7} \widetilde{\nu}(1 / h)$. Из (2.8) и (2.11) следует (2.3).

Оценка сверху в (2.4) и соотношение

$$
h^{3} \mathbf{E} X^{3}(h) \leqslant c_{8} \kappa\left(\frac{1}{h}\right)
$$

проверяются при помощи (2.3) аналогично (2.11). Кроме того,

$$
e^{-1} \widehat{\nu}\left(\frac{1}{h}\right) \leqslant h \mathbf{E} X(h) L(h)
$$

и, следовательно (см. также (2.7)),

$$
c_{3} \kappa\left(\frac{1}{h}\right) \leqslant h \mathbf{E} X(h) \leqslant c_{4} \kappa\left(\frac{1}{h}\right) \leqslant c_{9} .
$$

Теперь предположим, что выполнено левое неравенство (1.9). Тогда

$$
V(y)-V(b y)=\int_{b y}^{y} \frac{d \nu(u)}{u} \geqslant \widehat{\nu}(y)\left(1-\frac{1}{c_{1}}\right), \quad y \leqslant y_{0} .
$$


Принимая во внимание $(2.13),(2.14)$ и [2, формула (2.9)], видим, что при всех достаточно больших $h$

$$
L(h) h^{2} \mathbf{D} X(h) \geqslant \widehat{\nu}\left(\frac{1}{h}\right),
$$

что с учетом (2.2) и (2.12) завершает проверку соотношений (2.3) и (2.4).

Соотношение (2.5) с учетом (2.14) обосновывается так же, как лемма 2 в [2]. Лемма 1 доказана.

Для проверки теоремы 2 воспользуемся рассуждениями из [3, теорема 2.3], применив в соответствующих местах подходящие оценки из леммы 1.

В доказательстве теоремы 3 ключевую роль играет следующий результат.

Лемма 2 (см. [4, теорема 3]). При всех $r \in(0, \mathbf{E} S) u \varepsilon>0$

$$
e^{-Q(\gamma)} \geqslant \mathbf{P}(S \leqslant r) \geqslant e^{-(1+2 \varepsilon) Q(\gamma)}\left(1-\left(\frac{1+\varepsilon}{\varepsilon} a(\gamma)\right)^{2}\right),
$$

где $\gamma=\gamma(r)$ является решением уравнения $m(\gamma)=r u$

$$
a(\gamma)=\sup _{h>\gamma} \frac{h \sigma(h)}{Q(h)} .
$$

Теорема 3 вытекает из леммы 2 при $\varepsilon=K\left(a^{2}(\gamma) / Q(\gamma)\right)^{1 / 3}$ (здесь $K-$ некоторая достаточно большая постоянная), если иметь в виду, что $Q(h), h \geqslant 0$, монотонно возрастает от 0 до $-\ln \mathbf{P}(S=0)=\infty$ и что $a(\gamma) Q(\gamma) \leqslant \sup _{h>\gamma} h \sigma(h) \leqslant c_{16}$ при условии (1.12).

Проверим замечание 1 . Пусть $\lambda(u), u \geqslant 1,-$ некоторая непрерывная невозрастающая функция такая, что $\lambda(j)=\lambda_{j}, j=1,2, \ldots$. Обозначим

$$
\begin{aligned}
\lambda^{-1}(x) & =\sup \{u: u \geqslant 1, \lambda(u) \geqslant x\}, \quad x \leqslant \lambda_{1} ; \\
g_{y}(u) & =\min (1, y \lambda(u)), \quad u \geqslant 1, \quad y \geqslant 0 .
\end{aligned}
$$

Функция $g_{y}(u)$ не возрастает с ростом $u$, и, следовательно, при любом целом $M \geqslant 1$

$$
J_{M}=\mathbf{E}\left(\int_{M}^{\infty} g_{X}(u) d u\right) \leqslant \sum_{j \geqslant M} \mathbf{E} g_{X}(j) \leqslant J_{M}+\mathbf{E} g_{X}(M) .
$$

Имеем

$$
\int_{M}^{\infty} g_{y}(u) d u=-\int_{M}^{\infty}(u-M) d g_{y}(u)=-y \int_{\lambda^{-1}\left(y^{-1} \wedge \lambda_{M}\right)}^{\infty}(u-M) d \lambda(u) .
$$

Но в обозначениях замечания 1

$$
-y \int_{\lambda^{-1}(1 / y)}^{\infty} u d \lambda(u) \asymp-y \int_{\lambda^{-1}(1 / y)}^{\infty} b\left(\frac{1}{\lambda(u)}\right) d \lambda(u)=\bar{b}(y), \quad y \rightarrow \infty .
$$

Отсюда и из $(2.15),(2.16)$ при $M=1$ следует (1.1).

Теперь проверим утверждение перед теоремой 3 . Пусть $u=c / \lambda_{k}$. Очевидно (см. $(1.2)$ и $(2.2))$, что

$$
\sigma^{2}(u)=\sum_{j \geqslant 1} \lambda_{j}^{2} \mathbf{D}\left(u \lambda_{j}\right)
$$

Поэтому $u^{2} \sigma^{2}(u) \geqslant c^{2} \mathbf{D} X(c)$, откуда $\lim \sup _{\gamma \rightarrow \infty} \gamma \sigma(\gamma)>0$.

Далее займемся замечанием 3. Будем предполагать, что индекс $N=N(h)$ при всех достаточно больших $h$ удовлетворяет условию

$$
h \lambda_{N}>1 \geqslant h \lambda_{N+1} .
$$


Имеем (см. (2.17))

$$
h^{2} \sigma^{2}(h)=\left(\sum_{j=1}^{N}+\sum_{j>N}\right) h^{2} \lambda_{j}^{2} \mathbf{D} X\left(h \lambda_{j}\right)=I_{1}+I_{2} .
$$

Из $[3$, лемма 3$]$ следует, что при условии $(\mathbf{F})$

$$
c_{3} \leqslant \frac{h^{2} \mathbf{D} X(h)}{G(h)} \leqslant c_{4}, \quad 0<h \leqslant 1,
$$

где $G(h)=\mathbf{E}(1 \wedge h X)^{2}$ (для оценки в сверху (2.20) условие $(\mathbf{F})$ не требуется).

Таким образом, с учетом (2.18) при любом целом $M \geqslant 1$

$$
I_{2} \geqslant c_{3} \sum_{N<j \leqslant N+M+1} G\left(h \lambda_{j}\right) \geqslant c_{3} M G\left(h \lambda_{N+M+1}\right) \geqslant c_{3} M\left(\frac{\lambda_{N+M+1}}{\lambda_{N}}\right)^{2} G(1),
$$

откуда при условии (1.13) следует (1.7).

Условие (1.13) вытекает из (1.14) и (1.15), поскольку в этом случае $g(N+M+$ 1) $-g(N+1) \leqslant M g(N+1) /(N+1)$.

В заключение рассмотрим замечание 4. Пусть выполнены условия (1.14) и (1.16). Вернемся к равенству (2.19). Имеем аналогично (2.15) (см. (2.20))

$$
I_{2} \leqslant c_{4} \sum_{j>N} G\left(h \lambda_{j}\right) \leqslant c_{17} \sum_{j>N} G\left(h e^{-g(j)}\right) \leqslant c_{17}\left(\mathbf{E} \int_{N+1}^{\infty} \bar{g}_{h X}(u) d u+\mathbf{E} \bar{g}_{h X}(N+1)\right),
$$

где $\bar{g}_{y}(u)=\min \left(1,(y \bar{\lambda}(u))^{2}\right), \bar{\lambda}(u)=e^{-g(u)}, y \geqslant 0, u \geqslant u_{0}$.

Отметим, что функция $\bar{\lambda}(u), u \geqslant u_{0}$, непрерывна и согласно (1.16) строго убывает. Обозначим $b(s)=\bar{\lambda}^{-1}(1 / s), s \geqslant 1 / \bar{\lambda}\left(u_{0}\right)$, где $\bar{\lambda}^{-1}(\cdot)$ - функция, обратная к $\bar{\lambda}(\cdot)$.

Так же, как ранее в (2.16) при $M=N+1$, получаем

$$
T=\int_{M}^{\infty} \bar{g}_{h y}(u) d u=-(h y)^{2} \int_{\bar{\lambda}^{-1}\left((h y)^{-1} \wedge \bar{\lambda}(M)\right)}^{\infty}(u-M) d \bar{\lambda}^{2}(u) .
$$

Делая здесь замену переменной $u=b(t h)$, найдем

$$
T=y^{2} \int_{y \vee(1 /(h \bar{\lambda}(M)))}^{\infty}(b(t h)-M) d\left(-t^{-2}\right), \quad M=b\left(\frac{1}{\bar{\lambda}(M)}\right) .
$$

При этом (см. (2.18) и (1.14))

$$
\frac{1}{h \bar{\lambda}(M)}=\frac{1}{h \lambda_{N+1}} \lambda_{N+1} e^{g(N+1)} \geqslant \delta=c_{18} .
$$

Условие (1.16), как несложно проверить, равносильно тому, что функция $\widehat{b}(u)=$ $b(u) / \ln u$ при $u \geqslant K$ не возрастает.

Отсюда и из неравенства $b(u v) \leqslant(\ln u+\ln v) \min (\widehat{b}(u), \widehat{b}(v)), u, v \geqslant K$, следует, что $b(u v)-b(v) \leqslant b(u), u, v \geqslant K$, и, в частности, при всех достаточно больших $h$

$$
b(t h)-b(\delta h) \leqslant b\left(\frac{t}{\delta} \vee K\right) .
$$

Из (2.23)-(2.25) получим при некотором $y_{0}$

$$
T \leqslant y^{2} \int_{y \vee \delta}^{\infty}(b(t h)-b(\delta)) d\left(-t^{-2}\right) \leqslant c_{19}\left(1+b(y) I\left[y \geqslant y_{0}\right]\right),
$$

откуда по $(2.22),(2.21)$ и $(2.24)$ с учетом замечания 1 найдем $\sup _{h \geqslant h_{0}} I_{2} \leqslant c_{20}(1+$ $\left.\mathbf{E} b(X) I\left[X \geqslant y_{0}\right]\right)<\infty$. Таким образом (см. (2.19)), условие (1.12) равносильно условию $\sup _{h \geqslant h_{0}} I_{1}<\infty$. 
Если выполнено условие (1.10), то (см. (2.1))

$$
\kappa(y) \asymp \widehat{\kappa}(y)=\frac{l(y)}{\widetilde{l}(y)}, \quad y \rightarrow 0,
$$

а если, кроме того, $\widetilde{l}\left(e^{-t}\right)$, как функция $t$, логарифмически выпукла на бесконечности, то

$$
\widehat{\kappa}(y)=-\left.\left(\ln \widetilde{l}\left(e^{-t}\right)\right)^{\prime}\right|_{t=-\ln y},
$$

откуда следует, что $\widehat{\kappa}(y)$ не убывает в некоторой положительной полуокрестности нуля.

Из сказанного и условий $(1.14),(2.18)$ с учетом (2.3) получим (см. (2.19))

$$
I_{1} \asymp \widetilde{I}_{N}=\sum_{j=1}^{N} \widehat{\kappa}\left(\frac{e^{g(j)}}{h}\right), \quad h \rightarrow \infty,
$$

причем

$$
\widetilde{J}_{N} \leqslant \widetilde{I}_{N} \leqslant \widetilde{J}_{N}+\widehat{\kappa}\left(\frac{e^{g(N)}}{h}\right)
$$

где $\widetilde{J}_{N}=\int_{1}^{N} \widehat{\kappa}\left(e^{g(u)} / h\right) d u$.

Положим (см. (1.16)) $\nu(t)=g^{-1}(t), \bar{\kappa}(t)=\widehat{\kappa}\left(e^{-t}\right), r=\ln h$. Принимая во внимание условия (2.18) и (1.14), получим

$$
\widetilde{J}_{N}=-\int_{r-g(N)}^{r-g(1)} \bar{\kappa}(t) d \nu(r-t) \leqslant\left(\int_{-c_{21}}^{r / 2}+\int_{r / 2}^{r-g(1)}\right) \bar{\kappa}(t) d(-\nu(r-t))=J_{1}+J_{2} .
$$

Очевидно (см. (1.16) и (2.26)), что функции $\bar{\kappa}(t)$ и $\nu(t) / t$ не возрастают на бесконечности. Предполагая без потери общности, что эти функции не возрастают при всех $t \geqslant 1$, найдем

$$
\begin{aligned}
& J_{2} \leqslant \bar{\kappa}\left(\frac{r}{2}\right) \nu\left(\frac{r}{2}\right) \leqslant \nu\left(\frac{r}{2}\right) \frac{2}{r} \int_{0}^{r / 2} \bar{\kappa}(u) d u \\
& J_{1} \leqslant \int_{-c_{21}}^{r / 2} \bar{\kappa}(u) d u \max _{u \in\left(r / 2, r+c_{21}\right)} \frac{\nu(u)}{u} \leqslant \frac{2}{r} \nu\left(\frac{r}{2}\right) \int_{-c_{21}}^{r / 2} \bar{\kappa}(u) d u .
\end{aligned}
$$

Имея в виду (2.19) и (2.27)-(2.29), несложно убедиться в том, что условие (1.12) следует из (1.17).

Проверим остальные утверждения замечания 4. Условие $g(u) / u^{K} \searrow$, очевидно, равносильно предположению $\nu(u) / u^{1 / K} \nearrow$, что вместе с (2.29) приводит к оценкам

$$
\widetilde{J}_{N} \geqslant-\int_{c_{22}}^{r / 2} \bar{\kappa}(t) d \nu(r-t) \geqslant \frac{2}{K r} \nu\left(\frac{r}{2}\right) \int_{c_{22}}^{r / 2} \bar{\kappa}(u) d u
$$

и (см. (2.19), (2.26)-(2.28) и (1.17)) $h^{2} \sigma^{2}(h) \geqslant I_{1} \geqslant c_{23} J(\nu(\ln h))$. Отсюда следуют нужные результаты. Замечание 4 полностью проверено.

\section{СПИСОК ЛИТЕРАТУРЫ}

1. Lifshits M. A. On the lower tail probabilities of some random series. - Ann. Probab., 1997, v. 25, № 1, p. 424-442.

2. Розовский Л. В. О вероятностях малых уклонений сумм независимых положительных случайных величин. - Зап. науч. сем. ПОМИ, 2007, т. 341, с. 151-167.

3. Розовский Л. В. Малые уклонения сумм независимых положительных случайных величин с общим распределением, убывающем в нуле не быстрее степени. - Зап. науч. сем. ПОМИ, 2007, т. 341, с. 151-167. 
4. Rozovsky L. V. Remarks on a link between Laplace transform and distribution function of a nonnegative random variable. - Statist. Probab. Lett., 2009, v. 79, № 13, p. 15011508.

5. Петров В. В. Предельные теоремы для сумм независимых случайных величин. М.: Наука, 1987, 320 с.

6. Lifshits M.A. Bibliography on small deviation probabilities. http://www.proba. jussieu.fr/pageperso/smalldev/biblio.pdf

Поступила в редакцию

28.I.2014

\title{
(C) 2015 г. ШАШКИН А. П.*
}

\section{ФУНКЦИОНАЛЬНАЯ ПРЕДЕЛЬНАЯ ТЕОРЕМА ДЛЯ ИНТЕГРАЛОВ ПО МНОЖЕСТВАМ УРОВНЯ ГАУССОВСКОГО СЛУЧАЙНОГО ПОЛЯ ${ }^{1)}$}

\begin{abstract}
Рассматриваются интегралы непрерывных случайных функций по случайным мерам, порожденным множествами уровня гауссовского случайного поля. Показано, что при определенных требованиях к порождающему гауссовскому полю такие интегралы образуют непрерывный случайный процесс, индексированный точками уровня. В ситуации, когда интегрируемое случайное поле удовлетворяет некоторому условию слабой зависимости, доказана функциональная центральная предельная теорема для построенных так случайных процессов.
\end{abstract}

Ключевые слова и фразы: гауссовские случайные поля, функциональная предельная теорема, множества уровня, мера Хаусдорфа, локальное время случайного поля, формула коплощади.

Геометрические структуры, порожденные непрерывными гауссовскими случайными полями, в последние десятилетия привлекают значительное внимание исследователей. Это связано как с их приложениями к моделированию пространственных структур в томографии и астрофизике, так и с интересом к новым теоретическим результатам, возникающим на стыке теории случайных полей и дифференциальной геометрии. Достаточно упомянуть книги [4], [5], а также обзор [11]. Начиная с классической формулы Райса для математического ожидания числа нулей гауссовского случайного процесса с непрерывно дифференцируемыми траекториями, было открыто много свойств геометрических характеристик множеств уровня, порожденных гауссовскими случайными полями. Особенно важную роль играет хаусдорфова мера множества уровня, которая тесно связана с площадью поверхности экскурсионного множества случайного поля и, таким образом, с теорией функционалов Минковского таких множеств. Для мер множества уровня известны явные выражения для второго и старших моментов (с условиями их конечности), различные формы центральной предельной теоремы. В недавних работах [3], [12] получены функциональные предельные теоремы в ситуации, когда рассматриваются одновременно все вещественные значения уровня и берутся хаусдорфовы меры каждого из соответствующих множеств уровня (наблюдаемых в некотором ограниченном окне, размер которого стремится к бесконечности).

В работе [10] установлен ряд предельных теорем для интегралов по множествам уровня непрерывного случайного поля на двумерной плоскости. Предполагалось, что

* Московский государственный университет им. М.В. Ломоносова, механикоматематический факультет, Москва, Россия; e-mail: ashashkin@hotmail.com

1) Работа выполнена при поддержке РФФИ (проект № 13-01-00612). 